Check for updates

Cite this: J. Mater. Chem. B, 2021 9, 8851

Received 16th July 2021,

Accepted 20th September 2021

DOI: $10.1039 / \mathrm{d} 1 \mathrm{tb} 01556 \mathrm{~g}$

rsc.li/materials-b

\section{High-throughput fabrication of high aspect ratio Ag/Al nanopillars for optical detection of biomarkers $\dagger$}

\author{
Ainash Garifullina (D) and Amy Q. Shen (D) *
}

\begin{abstract}
Nanomaterial-based optical techniques for biomarker detection have garnered tremendous attention from the nanofabrication community due to their high precision and enhanced limit of detection (LoD) features. These nanomaterials are highly responsive to local refractive index (RI) fluctuations, and their RI unit sensitivity can be tuned by varying the chemical composition, geometry, and dimensions of the utilized nanostructures. To improve the sensitivity and LoD values of these nanomaterials, it is common to increase both dimensions and aspect ratios of the fabricated nanostructures. However, limited by the complexity, prolonged duration, and elevated costs of the available nanofabrication techniques, mass production of these nanostructures remains challenging. To address not only high accuracy, but also speed and production effectiveness in these nanostructures' fabrication, our work reports, for the first time, a fast, high-throughput, and cost-effective nanofabrication protocol for routine manufacturing of polymer-based nanostructures with high sensitivity and calculated LOD in the pM range by utilizing anodized aluminum oxide (AAO) membranes as templates. Specifically, our developed platform consists of arrays of nearly uniform polystyrene nanopillars with an average diameter of $\sim 185 \mathrm{~nm}$ and aspect ratio of $\sim 11$. We demonstrate that these nanostructures can be produced at a high speed and a notably low price, and that they can be efficiently applied for biosensing purposes after being coated with aluminum-doped silver (Ag/Al) thin films. Our platform successfully detected very low concentrations of human $\mathrm{C}$-reactive protein (hCRP) and SARS-CoV-2 spike protein biomarkers in human plasma samples with LoDs of 11 and $5 \mathrm{pM}$, respectively. These results open new opportunities for day-to-day fabrication of high aspect ratio arrays of nanopillars that can be used as a base for nanoplasmonic sensors with competitive LoD values. This, in turn, contributes to the development of point-of-care devices and further improvement of the existing nanofabrication techniques, thereby enriching the fields of pharmacology, clinical analysis, and diagnostics.
\end{abstract}

\section{Introduction}

Motivated by the strong need for fast and accurate disease diagnostics, especially in light of the recent COVID-19 viral outbreak, light-matter interaction-based techniques for biomarker detection have gained momentum due to their high precision, real-time monitoring, accessibility, and simple-to-use features. ${ }^{1,2}$ These optical techniques commonly utilize nanostructures of certain chemical composition, size, or geometry, all of which can be controlled during the nanofabrication process. ${ }^{3-6}$ This led to the growing demand in development as well as improvement of high-throughput, fast, and affordable nanofabrication strategies.

Micro/Bio/Nanofluidics Unit, Okinawa Institute of Science and Technology Graduate University, 1919-1 Tancha, Onna-son, Okinawa 904-0412, Japan.

E-mail: ainash.garifullina@oist.jp, amy.shen@oist.jp

$\dagger$ Electronic supplementary information (ESI) available. See DOI: 10.1039/d1tb01556g
Current approaches used for improvement of the surface sensitivities of optical nanomaterials towards biomarkers are mainly based on using high performance chemical materials and increasing the size and aspect ratio of the base nanostructures. ${ }^{3,7-10}$ The first strategy exploits plasmonic materials with a smaller imaginary and a larger absolute value of the negative real parts of the dielectric constant such as silver (Ag). However, due to its high reactivity, $\mathrm{Ag}$ has not been actively used for detection of biomarkers. ${ }^{7,11}$ Nonetheless, recently it has been shown that doping thin films of Ag with aluminum $(\mathrm{Ag} / \mathrm{Al})$ enhances the stability of $\mathrm{Ag}$ films under ambient conditions and improves their sensitivity towards changes in the surrounding media. ${ }^{9,12}$ An additional, and arguably a more efficient, surface sensitivity enhancement strategy involves increasing the overall size and aspect ratio (length/width) of the base nanostructures. ${ }^{13-15}$ However, despite the significantly boosted surface sensitivity generated by these geometrical 
modifications, the complex, time-consuming, and expensive manufacturing process of such nanostructures makes the fabrication of these platforms challenging. ${ }^{16}$ Existing nanofabrication techniques can be divided into two main groups: high-resolution but lowthroughput and high-throughput but low-resolution methods. ${ }^{17,18}$ Production of nanostructures in high-resolution primarily involves techniques such as electron beam, X-ray, soft, photon-beam, topdown, and nanoimprint lithography techniques, all of which involve intense amounts of nanofabrication time and high quality, expensive chemicals. ${ }^{16,17,19,20}$ The lithography-based techniques are high-resolution but less high-throughput when compared to solely wet chemical synthesis-based methods, which can be efficiently used to fabricate nanostructures over large areas but in decreased resolution. ${ }^{21}$

In this study, we report a fast, cost-effective, and highthroughput protocol for fabrication of high aspect ratio plasmonic nanopillars with great reproducibility. To speed up the nanofabrication process, whilst simultaneously notably decreasing the production cost, we used commercially available anodized aluminum oxide (AAO) membranes as templates for shaping the top surface of the polystyrene polymer film into nanopillars of the preferred dimensions, with a resulting average diameter of $\sim 185 \mathrm{~nm}$ and aspect ratio of $\sim 11$. Next, we coated these nanopillars with thin films of aluminum-doped silver $(\mathrm{Ag} / \mathrm{Al})$ and successfully applied our developed platform for the detection of biomarkers through standard immunoassay experiments with both human C-reactive protein (hCRP) and SARS-CoV-2 antibodyantigen systems. We then confirmed the biosensing efficacy of the resulting nanomaterials by comparing immunoassay results and calculated their limits of detection (LoD) in the PBS buffer solution to be 10 and $4 \mathrm{pM}$ for hCRP and SARS-CoV-2 spike protein antigens, respectively. Finally, to validate our nanomaterial performance in real samples, we conducted the same antigen detection experiments in spiked human plasma samples and found the LoD to be on the same orders of magnitude, 11 and $5 \mathrm{pM}$, respectively.

\section{Chemicals and materials}

Anodized aluminum oxide membranes (diameter $=13 \mathrm{~mm}$, thickness $=200 \mu \mathrm{m}$, and nanopore size $=200 \mathrm{~nm}$ ) were acquired from Global Life Science Technologies, Japan. A polydimethylsiloxane (PDMS) elastomer kit was purchased from Dow Sylgard 184 , USA. Glycerol $\left(\mathrm{C}_{3} \mathrm{H}_{8} \mathrm{O}_{3}, \geq 99.5 \%\right)$, acetone $\left(\left(\mathrm{CH}_{3}\right)_{2} \mathrm{CO}\right.$, $\geq 99 \%$ ), tert-butyl alcohol (TBA, $\left(\mathrm{CH}_{3}\right)_{3} \mathrm{COH}, \geq 98 \%$ ), ethanol $\left(\mathrm{C}_{2} \mathrm{H}_{5} \mathrm{OH}, \geq 98 \%\right)$, polystyrene (PS) film $\left(\left(\mathrm{C}_{8} \mathrm{H}_{8}\right)_{n}\right.$, thickness = $190 \mu \mathrm{m}, \mathrm{GF} 48641876)$, 11-mercaptoundecanoic acid (MUA, 450561, $\geq 98 \%$ ), 6-mercapto-1-hexanol ( $\mathrm{MCH}, 725226$, $\geq 97 \%$ ), Tween 20, bovine serum albumin (BSA, A2153, $\geq 96 \%$ ), phosphate-buffered saline tablets (PBS, P4417), and human plasma were all purchased from Sigma-Aldrich, Japan. 1-Ethyl3-(3-(dimethyl-amino)propyl)carbodiimide hydrochloride (EDC, 22980), $N$-hydroxy-succinimide (NHS, 24500, $\geq 98 \%$ ), and interleukin 6 (IL-6, PHC0066) were purchased from Thermo Scientific, Japan. Deionized (DI) water from an $18.2 \mathrm{M} \Omega \mathrm{cm}^{-1}$ Milli-Q Integral 3 water purification system (Millipore, Germany) was used for rinsing and preparing buffer solutions. Human C-reactive protein (hCRP, MAB17072-SP, $\geq 99 \%$ ) and human CRP antibody (anti-hCRP, MAB17072-100) were purchased from R\&D Systems, Japan. The SARS-CoV-2 spike protein peptide (ABIN1382273) and SARS-CoV-2 spike protein antibody (ABIN1030641) were purchased from antibodies-online.com, Germany.

\section{Results and discussion}

\subsection{Plasmonic polystyrene nanopillars with controlled aspect ratio}

Our fast, simple, and versatile protocol for fabrication of the optically active polymer-based nanopillars with controlled dimensions and aspect ratios involves three key steps: preparation of the template for further shaping of the polystyrene film surface into polystyrene nanopillars, fabrication of the polystyrene nanopillars using the prepared template, and plasmonic metal deposition on the surface of the formed polystyrene nanopillars.

3.1.1 Fabrication of the PDMS-filled AAO templates. High aspect ratio polystyrene (PS) nanopillars were fabricated following a modified protocol of the Feng research group. ${ }^{22}$ In order to use anodized aluminum oxide (AAO) membranes for shaping PS nanopillars, we needed to prepare polydimethylsiloxane (PDMS)-filled AAO templates first. Although AAO membranes have the necessary architecture to produce uniform polymer nanopillars, because of the elevated adhesion properties of aluminum oxide, extraction of PS nanopillars from the AAO membranes directly resulted in stretching or breaking of the polymer nanostructures. ${ }^{23}$ Thus, pre-coating the AAO nanopore surface with thin layers of the PDMS mixture not only helped to equalize the surface energies of the PDMS-coated AAO nanopores and the PS polymer, but also acted as a releasing agent for the PS nanopillars. ${ }^{22}$

PDMS-filled AAO templates, required for the next fabrication step, were prepared by first attaching the AAO membranes (nanopore diameter $=200 \mathrm{~nm}$, membrane thickness $=$ $200 \mu \mathrm{m})$ to the surface of the $20 \times 20 \mathrm{~mm}^{2} \mathrm{SiO}_{2}$ glass slide using a double-sided tape (thickness $=68 \mu \mathrm{m}, 3 \mathrm{M}, 8018 \mathrm{PT}$ ) and then drop-casting $15 \mu \mathrm{L}$ of the PDMS and curing agent mixture in a $35: 1$ ratio by weight (Fig. 1a(i)). Although the recommended ratio of the PDMS to its curing agent is $10: 1$, for our purposes the $35: 1$ ratio worked notably better. Decreasing the density and viscosity of the PDMS mixture accelerated its penetration inside the AAO nanopores. In addition, since in the later steps pressure was applied on top of the PDMS-coated AAO membranes, decreasing the modulus of the final cured PDMS by using less curing agent prevented the delicate AAO templates from being damaged. Spin-coating a small volume of the uncured PDMS mixture at a high speed $(5000 \mathrm{rpm}$, 60 seconds) ensured uniform spreading of the liquid PDMS mixture layer on the AAO membrane surface (Fig. 1a(ii)), leading to the filling of the interiors of these AAO membranes' nanopores with the uncured PDMS:curing agent mixture. After spincoating the PDMS mixture, the PDMS-coated AAO membranes 
a) Fabrication of AAO templates:

i)

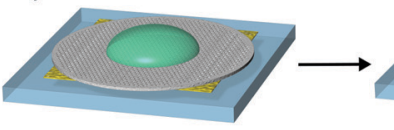

Drop-casting PDMS mixture on the AAO membrane

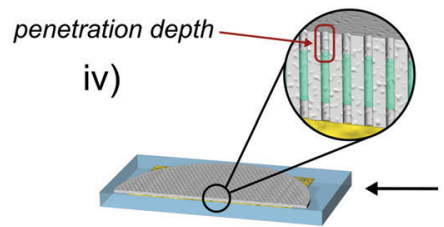

Dissolving extra PDMS mixture in TBA at $80^{\circ} \mathrm{C}$

glass substrate PDMS mixture double-sided tape $\square$ PS film

AAO membrane ii)

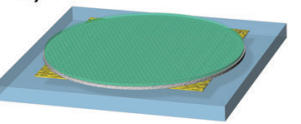

Spin-coating for 1 minute at 5000 RPM

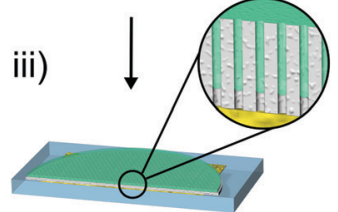

Degassing for 2 hours

b) Fabrication of PS nanopillars:

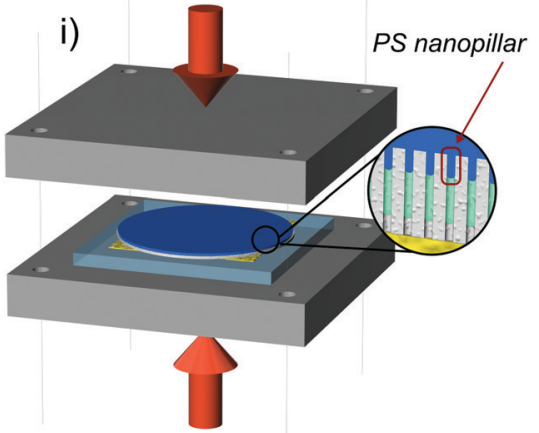

Melting PS films into the PDMS-coated AAO templates under $0.2 \mathrm{MPa}$ pressure at $100^{\circ} \mathrm{C}$

ii)

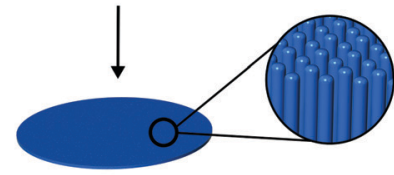

Deattaching PS film while flipping it $180^{\circ}$

c) Plasmonic coating of PS Nanopillars:

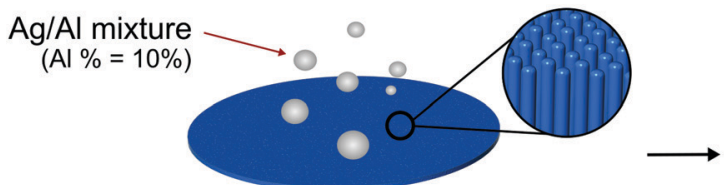

Co-sputtering $\mathrm{Ag}$ and $\mathrm{Al}$ metals at $200 \mathrm{~W}$ and $0.1 \mathrm{~nm} / \mathrm{s}$ and $160 \mathrm{~W}$ and $0.01 \mathrm{~nm} / \mathrm{s}$, respectively

PS nanopillar diameter $\sim 185 \mathrm{~nm}$ PS nanopillar height $\sim 2 \mu \mathrm{m}$

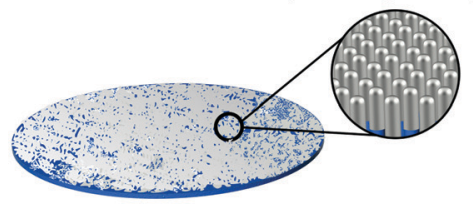

Ag/Al-coated PS nanopillars

d) SEM images of Ag/Al-coated PS Nanopillars:
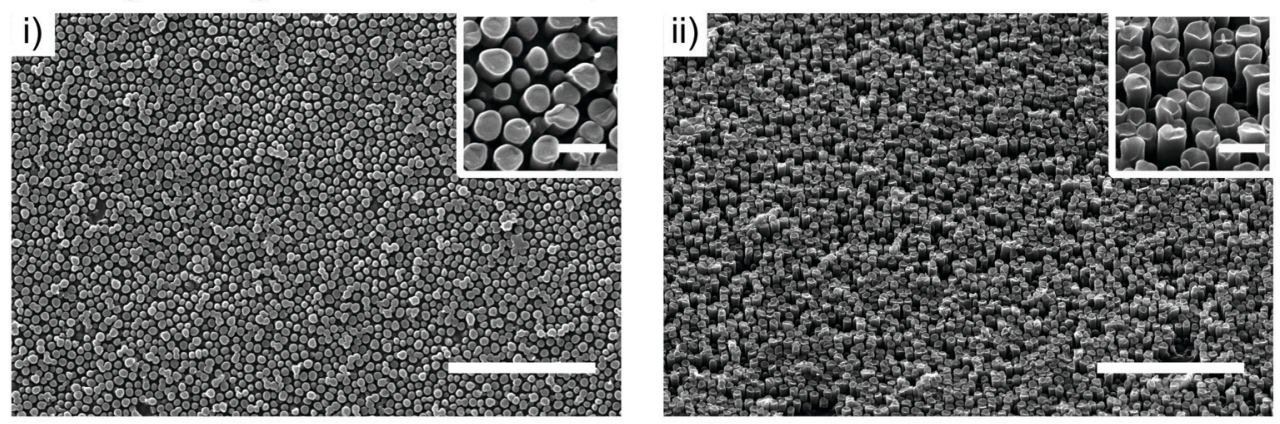

Fig. 1 (a) Fabrication of the PDMS-coated AAO templates: (i) a $15 \mu \mathrm{L}$ PDMS mixture (mint-green) was drop-casted on the AAO membrane (gray); (ii) the PDMS mixture was spin-coated on top of the AAO membrane at 5000 rpm for 60 seconds; (iii) the PDMS mixture-coated AAO membrane was degassed for 2 hours and, after the PDMS mixture infiltrated the AAO membrane nanopores, excess PDMS was dissolved in TBA at $80{ }^{\circ} \mathrm{C}$ for 4 minutes; (iv) the postTBA treated AAO membrane was cured overnight at $100{ }^{\circ} \mathrm{C}$ and thus the PDMS-filled AAO template was obtained. (b) Fabrication of the PS nanopillars: (i) the PS film (dark blue) pressed down onto the PDMS-filled AAO template with $0.2 \mathrm{MPa}$ pressure at $100{ }^{\circ} \mathrm{C}$ for 4 hours using a custom built aluminum mold (dark-gray); (ii) the resulting PS nanopillars on the surface of the PS film after peeling it off from the PDMS-filled AAO template. (c) Plasmonic coating of PS nanopillars with a mixture of Ag and Al (10 wt\%) metals. (d) SEM images of the Ag/Al-coated PS nanopillars: (i) top view and (ii) side view (tilt angle $=46^{\circ}$ ), scale bar $=5 \mu \mathrm{m}$, inset scale bar $=500 \mathrm{~nm}$.

were degassed for 2 hours at $-0.06 \mathrm{MPa}$ pressure using an AS ONE vacuum desiccator. Subsequent prolonged degassing of the PDMS-coated AAO membranes accelerated both capillarityand gravity-driven infiltration of the uncured PDMS mixture inside the nanopores of the membranes and eliminated air bubbles trapped under the PDMS mixture (see the magnified image in Fig. 1a(iii)). After the PDMS mixture infiltrated the nanopores, the PDMS-coated AAO membranes were immersed horizontally into $150 \mathrm{~mL}$ of the tert-butyl alcohol (TBA) solvent at an elevated temperature $\left(80^{\circ} \mathrm{C}\right)$ for fixed periods of time 
(1, 2, 3, 4, and 5 minutes), together with a magnetic bar being stirred at $350 \mathrm{rpm}$. In this step, the hot TBA solvent dissolved any excess amounts of the uncured PDMS : curing agent mixture from the surface of the AAO membrane and slightly penetrated the interiors of the nanopores. Depth of this penetration depended on the total immersion time of the PDMS-coated AAO membranes into the hot TBA solvent (Fig. 1a(iv), where the upper gray part of the nanopores represents the interior of the PDMScoated membrane penetrated by the hot TBA solvent). The longer the PDMS-coated AAO membranes were immersed into the hot TBA solvent, the larger the amount of the PDMS: curing agent mixture was dissolved, leading to deeper resulting PDMS-coated nanopores (Fig. 1a(iv)). The penetration depth of these PDMScoated nanopores set the length of the later fabricated PS nanopillars (Fig. 1b(i)). Since the PDMS : curing agent mixture dissolution time was fixed, post-TBA polymer-coated AAO membranes had the same nanopore depth, leading to the PS nanopillars with the same aspect ratio (Fig. 2b). PDMS-filled AAO templates were formed after overnight curing of the post-TBA PDMS-coated AAO membranes at $100{ }^{\circ} \mathrm{C}$ and were used in the next fabrication step after being sonicated in ethanol at $25{ }^{\circ} \mathrm{C}$ for 5 minutes.

3.1.2 Fabrication of the polystyrene (PS) nanopillars. The prepared PDMS-filled AAO templates (Fig. 1a(iv)) were next used to produce arrays of the PS nanopillars (Fig. 1b) with well controlled dimensions and aspect ratios. Thin circular films of the PS polymer $($ diameter $=15 \mathrm{~mm}$, thickness $=190 \mu \mathrm{m}$ ) were cut from the PS film purchased from Sigma-Aldrich, Japan (GF48641867, $30 \times 30 \mathrm{~cm}^{2}$, thickness $\left.=190 \mu \mathrm{m}\right)$ using a Graphtec FC 2250-120 flatbed cutting plotter (cutting force = $50 \mathrm{fg}$, passes $=3$, speed $=5 \mathrm{~cm} \mathrm{~s}^{-1}$, and acceleration $=1 \mathrm{~cm} \mathrm{~s}^{-2}$ ). After cutting out the PS circles, these PS circles were continuously pressed on top of the PDMS-filled AAO templates with $0.2 \mathrm{MPa}$ pressure at the glass transition temperature of the PS $\left(T_{\mathrm{g}}=100{ }^{\circ} \mathrm{C}\right)$ for 4 hours. A uniform pressure of $0.2 \mathrm{MPa}$ was applied with compressive forces by clamping the top and bottom parts of a custom built aluminum mold and securing them with M6 bolts (the aluminum mold is shown in dark-gray in Fig. 1b(i)). A glass slide with the PDMS-filled AAO membrane was sandwiched between the top and bottom parts of the aluminum mold, which were then tightened with a torque screwdriver until the torque reached $120 \mathrm{cN} \mathrm{m}$. This setup was then cooled down to room temperature, and the reshaped PS films were carefully peeled off from the surface of the AAO templates, repeatedly rinsed with ethanol, and carefully blowdried with a weak stream of compressed $\mathrm{N}_{2}$ gas (Fig. 1b(ii)). Using higher temperatures while simultaneously applying pressure $(120 \mathrm{cN}$ m torque over the AAO template with a known diameter of $13 \mathrm{~mm}$ ) promoted melting and subsequent infiltration of the PS polymer inside the empty parts of the AAO template nanopores (gray empty regions on the top of the PDMS-filled AAO membranes in Fig. 1a(i) were filled with the blue PS polymer in Fig. 1b(i and ii)). Thus, controlled depth of the PDMS-filled AAO template nanopores $(\sim 150 \mathrm{~nm}$ to $2.42 \mu \mathrm{m})$, achieved using the fixed PDMS : curing agent mixture dissolution time in the hot TBA solvent, led to a sufficiently uniform height of the resulting PS nanopillars. Based on the SEM characterization
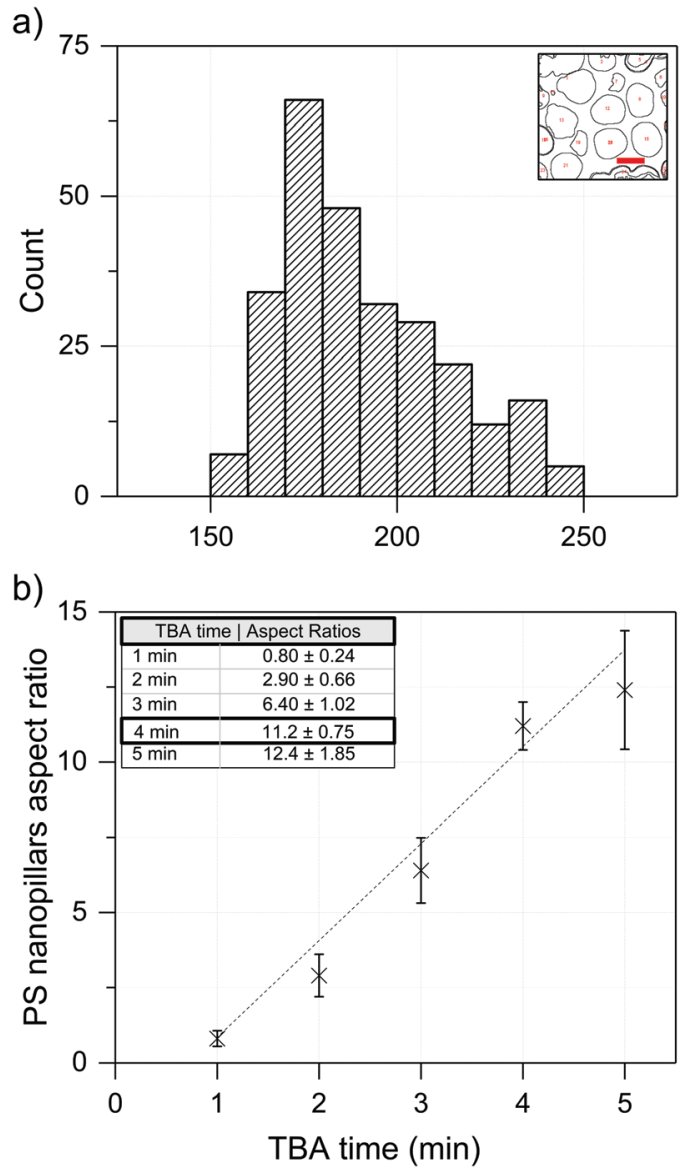

Fig. 2 (a) Size distribution histogram: average diameter of the polystyrene nanopillar $=185 \pm 22 \mathrm{~nm}$ (inset: sample particle diameter analysis using the ImageJ software, scale bar $=200 \mathrm{~nm}$ ). (b) Relationship between the aspect ratio of the PS nanopillars and the $80{ }^{\circ} \mathrm{C}$ TBA solvent contact time of the PDMS-coated AAO membranes: a longer TBA time led to a higher aspect ratio of the produced PS nanopillars (each data point is an average value with the standard deviation from 5 measurements).

(FEI Quanta 250 FEG, Fig. 1d), the PS nanopillars were almost uniformly distributed across the whole PS film surface. Small deviations in the average diameter of the PS nanopillars can be explained by the standard deviation of the nanopore diameter in the used AAO membranes. Using these SEM images, we measured the average PS nanopillar dimensions (average diameter $=185 \pm$ $22 \mathrm{~nm}$, Fig. 2a) and found that they have an increasing aspect ratio (height over diameter of the nanopillar) with increasing TBA contact time (Fig. 2b). Although the longer contact time with the hot TBA solvent led to a higher aspect ratio of the PS nanopillars ( 5 minutes -12.5 aspect ratio), the optimal hot TBA contact time was fixed at 4 minutes (height $\sim 2 \mu \mathrm{m}$ ) to avoid gravity-driven collapse of the PS nanopillars when a hot TBA dissolution time longer than 4 minutes was used. In addition to the prominent collapse of the PS nanopillars, immersing PDMS-coated AAO membranes in the hot TBA for over 4 minutes led to thinner layers of the PDMS coating on the inner sides of the nanopores, thereby increasing the adhesive forces between the AAO template and the later introduced polymer film (PS), thereby making extraction of the PS nanopillars less efficient. ${ }^{22}$ Generally, higher 
aspect ratio plasmonic nanopillars were expected to result in higher refractive index unit sensitivity and, consequently, better LoD values. However, the increased instability of polymer nanopillars with a 12.5 aspect ratio led to less reproducible refractive index measurements, thereby making systematic studies on this platform less reliable. As a result, we fixed the hot TBA contact time at 4 minutes, which resulted in PS nanopillars with an aspect ratio equal to $\sim 11.2(2.09 \mu \mathrm{m}$ height/185 $\mathrm{nm}$ diameter). After peeling the PS films off the surface of the AAO templates, the PDMS-filled AAO templates were successfully reused for fabrication of more PS nanopillar samples with highly reproducible results up to five times (Fig. S1 and S2, ESI $\dagger$ ).

Overall, our proposed protocol for the fabrication of high aspect ratio polymeric nanopillars is both cost- and timeeffective since it only requires a hot plate and takes slightly over 4 hours to produce a circular sample of a $13 \mathrm{~mm}$ diameter. The final size of the sensor platform is pre-defined by the size of the AAO membrane diameter, which can be larger or smaller if needed, making this protocol highly scalable. Meanwhile, other fabrication approaches require expensive cleanroom equipment and could take up to 20 hours (based on estimations using the electron-beam lithography manual provided by ELIONIX Inc.) to produce a sample of similar size containing nanostructures of the same dimensions (most of the nanopillarlike structures reported in the literature were fabricated by means of electron-beam lithography and had aspect ratios of $\leq 10) .{ }^{24-26}$ In addition, a broad selection range of various diameters, thicknesses, and nanopore diameters of the commercially available AAO membranes makes this fabrication protocol extremely tunable. Therefore, our protocol can be employed for highthroughput fabrication of the PS polymer (or a different polymer given that the protocol is properly optimized) nanopillars with controlled dimensions and aspect ratios in a quick, cost-effective, and reproducible manner.

3.1.3 Coating PS nanopillars with Al-doped Ag films $(\mathbf{A g} / \mathbf{A l})$. In this part of our work, we tested if the developed platform can be efficiently used for optical detection of biomarkers in both PBS buffer and diluted human plasma solutions. One example of these optical techniques is localized surface plasmon resonance (LSPR) spectroscopy, which is a highly efficient optical tool for identifying and quantifying biomolecules specific to certain diseases in a quick and labelfree manner. ${ }^{27-29}$ The LSPR phenomenon occurs when plasmonic nanostructures are smaller than the wavelength of the incident light they are exposed to, ${ }^{30,31}$ which leads to resonant electrons oscillating at the frequency affected by the shape, size, and chemical composition of these nanostructures, as well as the dielectric constant or refractive index (RI) of the medium around them. ${ }^{32,33}$ To make the resulting PS polymer nanopillars optically active, we coated them with thin films (thickness $=20 \mathrm{~nm}$ ) of a plasmonic material using an atomic e-beam evaporator (PLASSYS Bestek, France) at $0.1 \mathrm{~nm} \mathrm{~s}^{-1}, 1 \times 10^{-7}$ Torr, and $80 \mathrm{~mA}$ while constantly rotating the sample to improve the uniformity of the deposited metal layer. Since gold ( $\mathrm{Au}$ ) is stable under ambient conditions, readily available, and is often used as a benchmark platform for plasmonic studies, we initially coated the PS nanopillars with thin films of pure atomic $\mathrm{Au}$ (Fig. 3a(i)). ${ }^{34}$ To eliminate additional LSPR effect contributions from individual plasmonic nanoislands, it was essential to coat the upper part of these nanopillars with continuous Au films. ${ }^{9}$ However, nanometer-scale dimensions of our nanostructures limited the maximum thickness of the deposited metal layer in order to avoid gravity-driven collapse of the PS nanopillars. Given that atomic Au starts forming nearly continuous films from 10 to 11 $\mathrm{nm},{ }^{35}$ PS polymer nanopillars were coated with $20 \mathrm{~nm}$ of $\mathrm{Au}$. Energy dispersive X-ray spectroscopy (EDS, JEOL JSM-7900F) analysis of the fabricated samples was performed using sputtering with small amounts of $\mathrm{Au}$ to reduce the charging effect from the materials' surfaces, which is why all of the recorded spectra were expected to possess small peaks corresponding to $\mathrm{Au}$. The EDS spectrum of the PS nanopillars after exposure to atomic Au (Fig. 3a(i)) demonstrated that the e-beam deposition of Au resulted in Au-coated PS nanopillars, whereas the UV-Vis spectrum of these nanopillars showed that they were plasmonically active with the main LSPR peak at $\sim 600 \mathrm{~nm}$ (Fig. $3 \mathrm{~b}$, red spectrum).

Refractive index unit sensitivity (RIS) of the Au-coated nanopillars was measured using five concentrations of aqueous glycerol solutions (Fig. 3c) corresponding to the RI values of 1.33 (water), 1.34 (10 wt\% glycerol), 1.36 (20 wt\% glycerol), 1.37 (30 wt\% glycerol), 1.38 (40 wt\% glycerol), and 1.40 (50 wt\% glycerol). The measured RIS for Au-coated PS nanopillars was found to be $229 \pm 7 \mathrm{~nm}$ per RIU, which is comparable to those for similar optical sensing nanomaterials available on the market. $^{36,37}$ Replacing the Au coating of the PS nanopillars with $20 \mathrm{~nm}$ thick films of Al-doped (10 wt\%) Ag (Ag/Al) led to an enhanced RIS value of $291 \pm 6 \mathrm{~nm}$ per RIU (Fig. 3c). The EDS analysis of $\mathrm{Ag} / \mathrm{Al}$ films demonstrated that co-sputtering of the $\mathrm{Ag}$ and $\mathrm{Al}$ metals led to Al-doped $\mathrm{Ag}$ thin films, which were observed as notable $\mathrm{Ag}$ and $\mathrm{Al}$ peaks (inset of Fig. 3a(ii)), implying that the surface of the PS nanopillars was coated with $\mathrm{Ag} / \mathrm{Al}$ thin films (small $\mathrm{Au}$ peaks were caused by the initial $\mathrm{Au}$ sputtering step for reducing the surface charging effect prior to imaging of the samples). To form thermally stable $\mathrm{Ag} / \mathrm{Al}$ nanofilms, we followed a protocol provided by Gu et al. In their work, Al-doped Ag nanofilms were formed via co-sputtering of $\mathrm{Al}$ and Ag metals (Fig. 1c). ${ }^{9}$ For our sample preparation, the sample holder was constantly rotated and $\mathrm{Al}$ was sputtered at a $160 \mathrm{~W}$ power and a $0.01 \mathrm{~nm} \mathrm{~s}^{-1}$ deposition rate, whereas $\mathrm{Ag}$ was deposited at $200 \mathrm{~W}$ and $0.1 \mathrm{~nm} \mathrm{~s}^{-1}$, respectively. This led to the formation of Al-doped $\mathrm{Ag}$ films that were chemically more stable and oxidation resistant due to spontaneous formation of a protective or so-called capping layer between $\mathrm{Al}$ atoms and atmospheric oxygen. The UV-Vis spectrum of these nanopillars showed the main LSPR peak at $\sim 490 \mathrm{~nm}$ (Fig. 3b, blue spectrum). In addition, both $\mathrm{Au}-$ and $\mathrm{Ag} / \mathrm{Al}$-coated plasmonic nanopillars displayed two distinct plasmon frequency peaks which are characteristic of plasmonic nanostructures with anisotropic shape, specifically of nanorod-like structures. ${ }^{4,24}$ The nanorod-like shape of these nanostructures provides two axes for plasmon oscillations: along the long axis - longitudinal resonance mode and along the shorter axis - transverse 
a) EDS spectra of plasmonic PS nanopillars:

i)
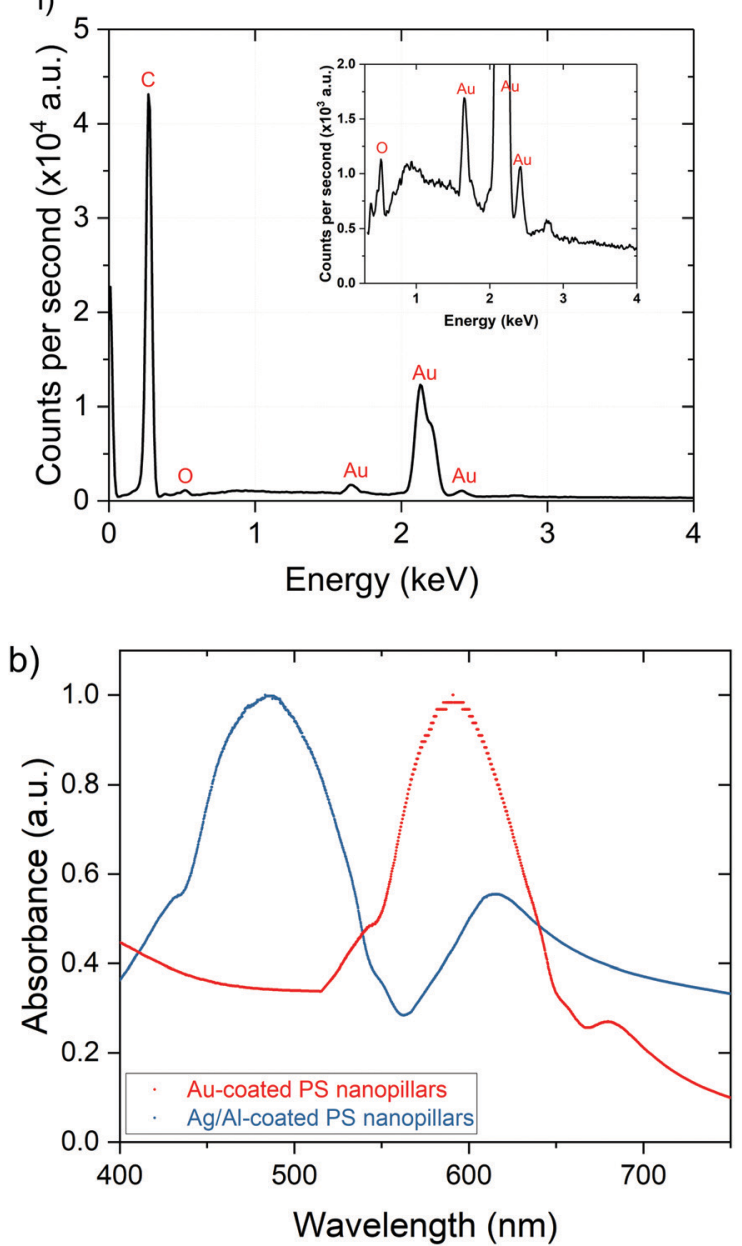

ii)
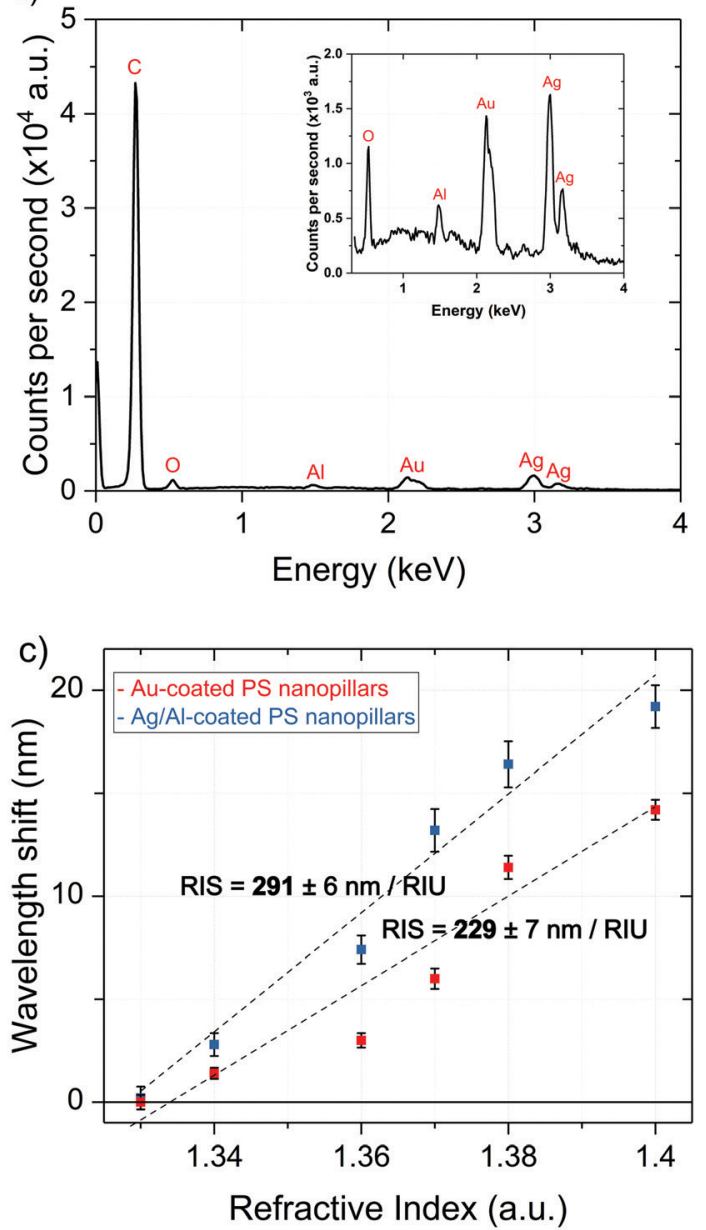

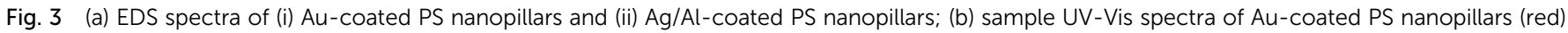

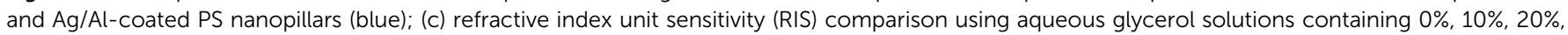

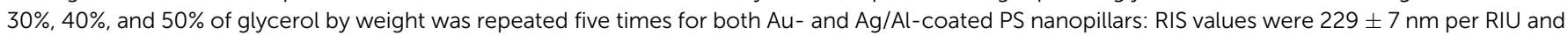
$291 \pm 6 \mathrm{~nm}$ per RIU, respectively.

resonance mode (Fig. 3b: larger peak to the left and smaller peak to the right, respectively). ${ }^{38}$ The longer LSPR wavelength of the transverse resonance modes provides additional information over existing single peak biomolecule detection techniques, such as detection of biomarkers in the near infrared region. This can be helpful for the analysis of bodily fluids (including blood, plasma, saliva, and urine) that commonly comprise biomolecules resonating in the near infrared region (thereby making their detection in the UV-Vis region challenging). ${ }^{39}$ Nonetheless, since it has been demonstrated that the transverse LSPR peaks are less sensitive to the dielectric constant fluctuations of the surrounding media when compared to the longitudinal ones, ${ }^{40,41}$ in the interest of this study, we primarily focused on the maximum peak values of the longitudinal LSPR modes.

3.1.4 Biosensing performance of high aspect ratio $\mathbf{~} \mathrm{g} / \mathrm{Al}$ coated PS nanopillars. The absorbance spectra of the material surfaces were measured in the $280-850 \mathrm{~nm}$ wavelength range by transmitting light through the sample functionalized with biomolecules and by comparing the collected signal against the reference (Fig. 4). Given that Ag/Al-coated PS nanopillars resulted in a higher RIS value, we proceeded with performing standardized immunoassay tests using the $\mathrm{Ag} / \mathrm{Al}$ nanoplasmonic platform for detection of changes in the UV-Vis spectra caused by biomolecular interaction events, and calculated the experimental LoD values for detection of the human C-reactive protein (hCRP) and of the severe acute respiratory syndrome coronavirus 2 (SARSCoV-2) spike protein. Regarding the two biomarkers used in the immunoassay tests, hCRP is a well-studied and thoroughly characterized acute phase protein that actively rises in response to an infection, inflammation, or any tissue injury; in addition, it is commonly used to predict cardiovascular diseases or to characterize metabolic syndrome, which makes its accurate detection essential for diagnostic purposes. ${ }^{42,43}$ SARS-CoV-2, on the other hand, is a recently discovered RNA virus that led to the 2019 coronavirus infectious disease (COVID-19) pandemic. ${ }^{44,45}$ SARSCoV-2's highly infectious nature, rapidness of spreading across the globe, and severity of the symptoms among the infected patients motivated the development of highly sensitive diagnostic 
a) Schematic of the UV-Vis measurement setup:
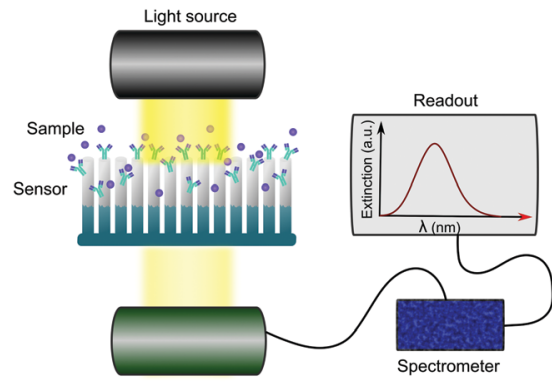

Detector

Fig. 4 (a) Schematic of the UV-Vis measurement setup: the tungsten halogen light source (HL-2000-HP-FHSA, Ocean Optics, Japan) emitted light on the material surface and the transmitted light was collected at the bottom using the detector, after which it was sent to the spectrometer (USB4000-UV-VIS-ES, Ocean Optics, Japan); the detected signal was visualized as a plot of maximum extinction (a.u.) versus wavelength $(\mathrm{nm})$ from 280 to $850 \mathrm{~nm}$ and analyzed using SpectraSuite spectrometer operating software (Ocean Optics, Japan); (b) real image of the UV-Vis measurement setup.

platforms for the detection of its antigens, ${ }^{46}$ which is why we used our developed nanoplasmonic platform to quantify one of its antigens, SARS-CoV-2 spike protein.

The biosensing capabilities of the PS nanopillars coated with $20 \mathrm{~nm}$ of $\mathrm{Ag} / \mathrm{Al}$ film were tested using aqueous hCRP and SARSCoV-2 spike protein antibody-antigen model systems. To monitor the changes occurring on the sensor surfaces, the UV-Vis spectra were measured before and after each surface chemistry step as well as after the introduction of biomarkers (Fig. 4). All antibody and antigen solutions were diluted in $0.01 \mathrm{M}$ phosphate-buffered saline (PBS) solution. Prior to the UV-Vis signal detection before and after each immunoassay step, all sensor surfaces were rinsed with wash buffer $(0.01 \mathrm{M}$ PBS $+0.05 \%$ Tween 20 detergent $)$ and carefully blow-dried with compressed $\mathrm{N}_{2}$ gas. Metal-coated PS nanopillars were functionalized with anti-hCRP and anti-SARSCoV-2 spike protein following an optimized MUA:MCH biomolecule immobilization protocol that was shown to be the most efficient for the immobilization of biomolecules on non-spherical plasmonic nanostructures. ${ }^{47}$ Briefly, these $\mathrm{Ag} / \mathrm{Al}$ nanopillars were incubated in a $1 \mathrm{M}$ : $9 \mathrm{M}$ solution of MUA: $\mathrm{MCH}$ diluted in ethanol ( $2 \mathrm{~mL}$, overnight, at $24{ }^{\circ} \mathrm{C}$, on a shaker), where the volume of the solution was pre-determined by the dimensions of the Petri dish that was used for the incubation step; here $2 \mathrm{~mL}$ was sufficient for complete immersion of the sensors. This was followed by 1 more hour of incubation in $2 \mathrm{~mL}$ of $1 \mathrm{mM} \mathrm{MCH}$ diluted in ethanol at $24{ }^{\circ} \mathrm{C}$ on a shaker. Due to spontaneous formation of covalent bonds between the $\mathrm{Ag}$ atoms and the thiol functional groups (-SH) of both MUA and MCH molecules, the introduced thiol linkers self-assembled on the metal coating of the PS nanopillars, and the ones that did not immobilize were rinsed with ethanol (Fig. 5a(i)). ${ }^{48,49}$ Later, the carboxyl $(-\mathrm{COOH})$ ends of the MUA linkers were activated by introducing $2 \mathrm{~mL}$ of aqueous EDC: NHS solution in a $4 \mathrm{M}: 1 \mathrm{M}$ ratio for $15 \mathrm{~min}$ at $24{ }^{\circ} \mathrm{C}$ on a shaker (Fig. 5a(ii)). Next, the aqueous EDC: NHS mixture was immedi-

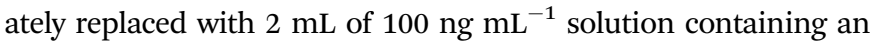

antibody of interest, anti-hCRP or anti-SARS-CoV-2 spike protein, diluted in 0.01 M PBS, and incubated for 2 hours at $4{ }^{\circ} \mathrm{C}$ on a shaker (Fig. 5a(iii)). Lastly, the functionalized platforms were rinsed with wash buffer, incubated in $2 \mathrm{~mL}$ of $0.01 \%$ bovine serum albumin (BSA) buffer for 1 hour at $24{ }^{\circ} \mathrm{C}$ on a shaker, rinsed with wash buffer again, and finally incubated in $2 \mathrm{~mL}$ of five different concentrations of hCRP and SARS-CoV-2 spike protein, respectively, for 1.5 hours at $4{ }^{\circ} \mathrm{C}$ on a shaker. More details on the immobilization protocol can be found in our previously published work. ${ }^{47}$ The UV-Vis spectra detected before and after the final antigen incubation step were used to evaluate the overall optical performance with respect to the specific antigen detection. Fig. 5b presents the UV-Vis spectra and averaged wavelength shifts measured after each immunoassay step when $\mathrm{Ag} / \mathrm{Al}$-coated PS nanopillars were used to detect $100 \mathrm{ng} \mathrm{mL}^{-1}$ solutions of hCRP and SARS-CoV-2 spike protein antigens. Specifically, the UV-Vis spectra plots in Fig. $5 \mathrm{~b}$ (i and iii) correspond to the capture of hCRP and SARS-CoV-2 spike protein, respectively. The wavelength shifts caused by the addition of MUA linkers and BSA blocking buffer were negligible, which can be explained by a relatively small size of the MUA molecules and by the lack of available space for BSA binding. The largest wavelength shifts were detected after immobilization of anti-hCRP and anti-SARSCoV-2 spike protein as well as after capture of their respective antigens (Fig. 5b(ii and iv), respectively).

The optical performance and LoD values of the fabricated nanoplasmonic platforms were estimated by plotting the detected wavelength shifts versus five different concentrations of hCRP and SARS-CoV-2 spike protein antigens diluted in both aqueous PBS and diluted human plasma solutions (Fig. 6). To ensure that the detected signals were caused by the antigens of interest rather than by non-specific biomarkers, we introduced a specificity test using interleukin-6 (IL-6) protein. Similar to hCRP, IL-6 is a well-characterized biomarker of immune system activation, which is why it suits well for the purposes of our specificity test. Initially, we measured $\Delta \lambda_{\text {LSPR }}$ caused by the control sample (0.01 M PBS) and a non-specific antigen sample (100 ng mL ${ }^{-}$IL-6 in 0.01 M PBS), and found that no notable wavelength shifts were caused by either of these samples, $\Delta \lambda_{\text {LSPR }} \sim 0 \mathrm{~nm}$ (inset of Fig. 6(a)). To quantitatively characterize the developed nanopillar platform, we calculated the LoD values using the wavelength shifts from Fig. 6 and the following formula: $\mathrm{LOD}=3.3 \times\left(\mathrm{SD}_{\text {blank }} /\right.$ slope $)$, where $\mathrm{SD}_{\text {blank }}$ is the standard error equal to the standard deviation calculated from the $0 \mathrm{ng} \mathrm{mL} \mathrm{m}^{-1}$ sample and the slope of the regression line (see detailed calculations in Section S2 in the ESI $\dagger) .{ }^{50}$ According to Fig. 6 and more detailed Fig. S4(a and b) (ESI $\dagger$ ), both hCRP and SARS-CoV-2 spike protein can be successfully detected in PBS using our platform with the respective LoD values of $1.2 \pm 0.36$

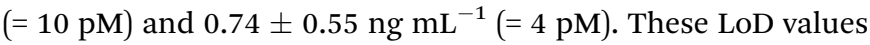
support the fact that the suggested protocol results in a highly sensitive platform composed of plasmonic nanopillars with regulated aspect ratios.

The real samples analyzed for clinical diagnostic purposes are significantly more complex than the aqueous PBS-based samples used for initial characterization. For this reason, we 


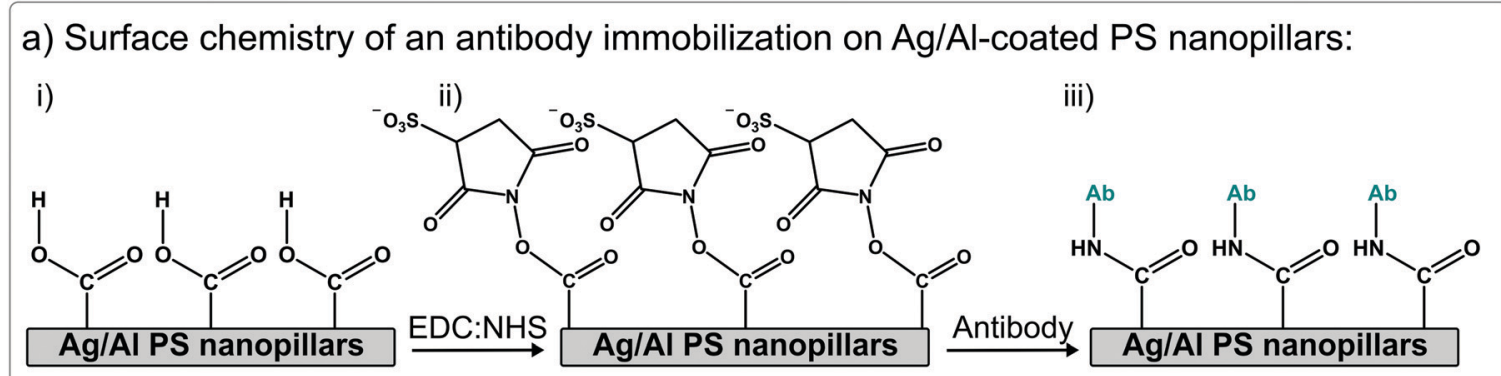

b) Wavelength shifts detected during the hCRP and SARS-CoV-2 immunoassay steps:
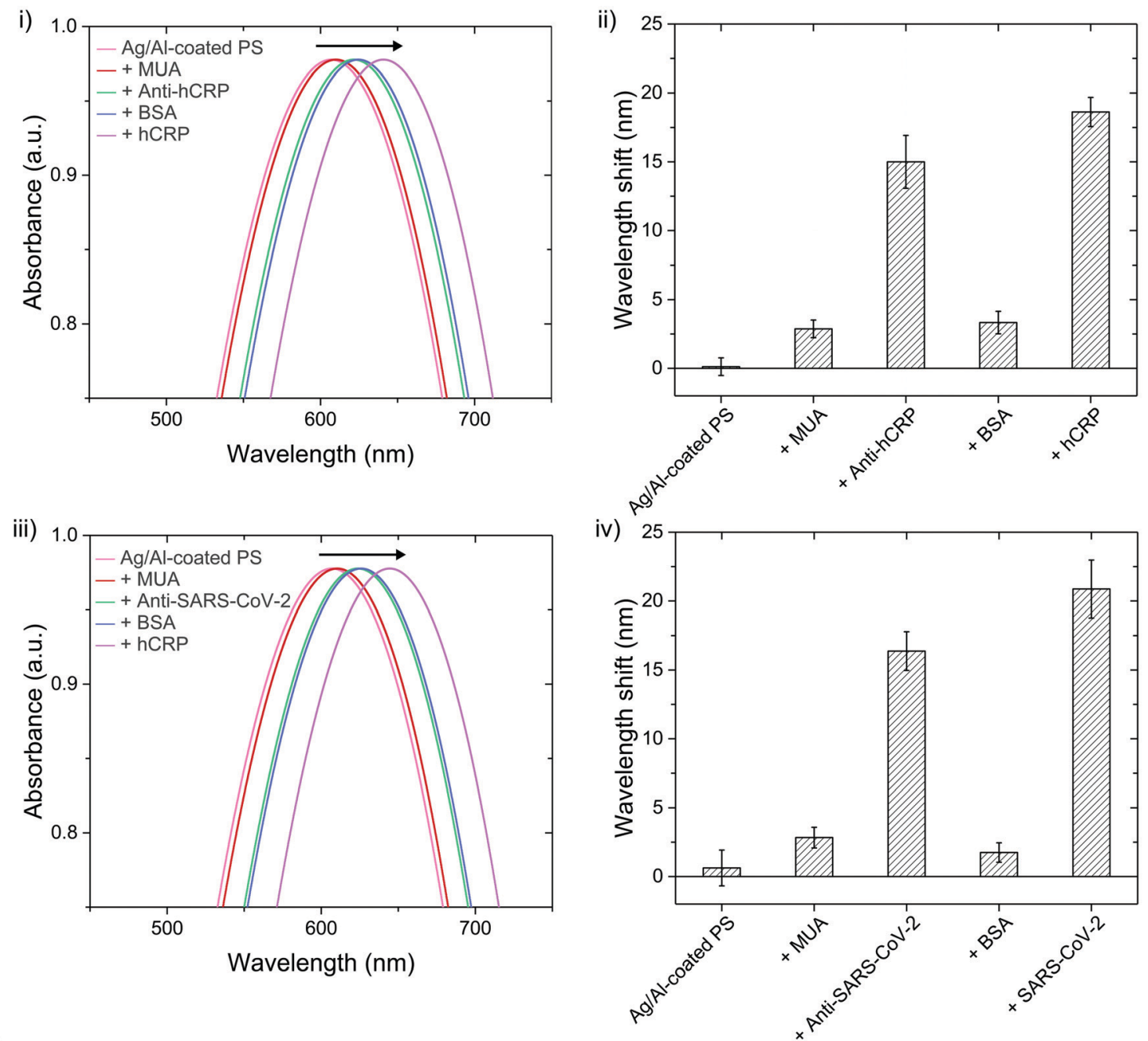

Fig. 5 (a) Antibody immobilization on the Ag/Al-coated PS nanopillars via carbodiimide crosslinker chemistry: (i) immobilization of MUA and MCH molecules via physical drop-casting, (ii) intermediate step after the addition of the EDC and NHS mixture: formation of a semi-stable amine-reactive NHS-ester, and (iii) formation of a stable amide bond after the addition of an antibody of interest. (b) $\Delta \lambda_{\text {LSPR }}$ detected during the immunoassay: (i) $\&$ (iii) UV-Vis spectra of Ag/Al-coated PS nanopillars measured after each immunoassay step using anti-hCRP/hCRP and anti-SARS-CoV-2/SARS-CoV-2 spike protein, respectively; (ii) $\&$ (iv) $\Delta \lambda_{\text {LSPR }}$ detected after each immunoassay step (with respect to the previous step) and averaged over 8 data points for anti-hCRP/hCRP and anti-SARS-CoV-2 spike protein/SARS-CoV-2 spike protein, respectively.

repeated the same antigen detection experiments with spiked human plasma samples $(1: 100$ in $0.01 \mathrm{M}$ PBS) instead of pure $0.01 \mathrm{M}$ PBS as a buffer. At this stage of the device development, to avoid extra scattering caused by using complex biological samples, we have diluted the human plasma samples with PBS buffer. $\Delta \lambda_{\text {LSPR }}$ caused by both control (diluted human plasma) and non-specific (100 $\mathrm{ng} \mathrm{mL}^{-1}$ IL-6 in diluted human plasma) samples were found to be negligible $(\sim 0 \mathrm{~nm})$, which ensured that the components of the human plasma did not interfere with our measurement system (Fig. 6b). The LoDs for hCRP and 

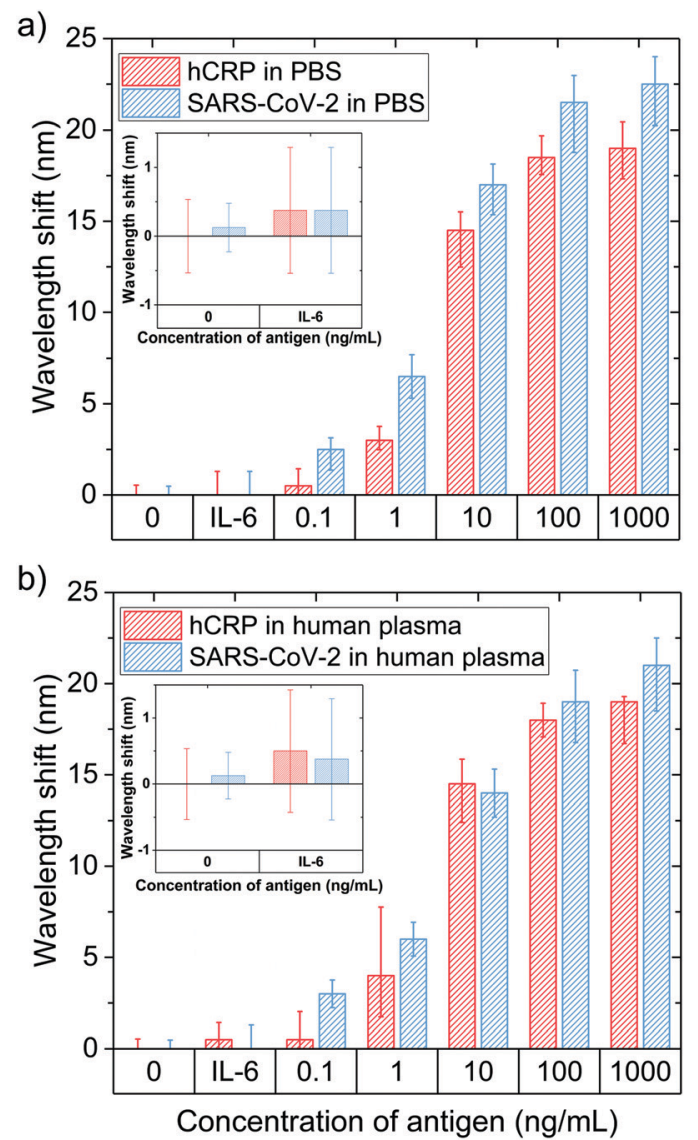

Fig. $6 \Delta \lambda_{\text {LSPR }}$ caused by the detection of $0,0.1,1,10,100$, and $1000 \mathrm{ng} \mathrm{mL}^{-1}$ hCRP \& SARS-CoV-2 spike protein, and non-specific interleukin-6 (IL-6) antigens diluted in (a) $0.01 \mathrm{M}$ PBS and (b) diluted human plasma with LoDs for hCRP equal to 10 and $11 \mathrm{pM}$ and those for SARS-CoV-2 spike protein equal to 4 and 5 pM in PBS and human plasma, respectively. Insets present the magnified plots of signals caused by the introduction of the control, $0 \mathrm{ng} \mathrm{mL}{ }^{-1}$ specific antigen, and of the non-specific antigen solution, $100 \mathrm{ng} \mathrm{mL}^{-1}$ IL-6; both solutions led to negligible wavelength shifts.

SARS-CoV-2 spike protein detection in the spiked human serum samples are comparable to those in pure PBS, $1.3 \pm 0.27$ (11 pM) and $0.93 \pm 0.57 \mathrm{ng} \mathrm{mL}^{-1}(5 \mathrm{pM})$, respectively. The calculated LoDs for our developed nanoplasmonic platforms in both PBS and human plasma are on the same orders of magnitude as those for other optical sensing platforms based on plasmonic nanopillarlike structures of similar dimensions and aspect ratios but fabricated using significantly more complex fabrication protocols (in terms of both time and cost). ${ }^{3,51}$ Overall, when compared with the LoDs of other biomarker detection methods used for quantification of SARS-CoV-2 biomarkers, ELISA (LOD = $100 \mathrm{ng} \mathrm{mL}^{-1}, \approx 0.5 \mathrm{nM}$ ) and quantum-dot based lateral flow assays (LoD $\left.=10 \mathrm{ng} \mathrm{mL}^{-1}, \approx 55 \mathrm{pM}\right),{ }^{52}$ our system exhibited 100 and 10-fold improvements of the LoD, respectively.

\section{Conclusions}

In this work, we developed a fast, high-throughput, low-cost, and highly reproducible protocol for the fabrication of polymerbased nanopillars with well-controlled dimensions and high aspect ratios. We demonstrated that, with plasmonic metal coating, this platform can be successfully used for biosensing purposes with enhanced RIS caused by replacing Au films with Al-doped Ag films. The final system successfully detected down to 11 and $5 \mathrm{pM}$ of hCRP and SARS-CoV-2 spike protein in spiked human plasma samples, respectively. In summary, (1) PDMSfilled AAO templates can be used for the fabrication of high aspect ratio PS nanopillars at high throughput and low cost, (2) Al-doped Ag films improve the sensitivity of plasmonic nanostructures towards fluctuations in the surrounding environment, and (3) the developed PS nanopillar-based system can be efficiently utilized for sandwich immunoassay-like detection of biomarkers in human plasma samples. The proposed nanofabrication protocol can be effectively optimized to a large variety of polymeric materials, nanoplatform dimensions (predefined by the diameter of the AAO membrane templates), and to various preferable dimensions of nanostructures (predefined by the geometry of the AAO membrane nanopores). All of these make our nanofabrication protocol highly advantageous for device integration and point-of-care optical sensing applications with a direct impact on fast and affordable clinical diagnostics.

\section{Author contributions}

A. G. and A. Q. S. conceived and designed the research project. A. G. carried out the experiments, generated and analyzed data, and wrote the original manuscript. A. Q. S. reviewed and revised the manuscript.

\section{Conflicts of interest}

There are no conflicts to declare.

\section{Acknowledgements}

The authors acknowledge the financial support from the Okinawa Institute of Science and Technology Graduate University (OIST) with subsidy funding from the Cabinet Office, Government of Japan. A. G. is a JSPS DC2 fellow (Japan Society for Promotion of Science) and this work is supported by JSPS KAKENHI (Grant No. 21J10403). The authors also thank Mr Kazumi Toda-Peters from the Micro/Bio/Nanofluidics Unit at OIST for useful discussions, assistance with experimental design of the project, and help with visuals and schematics of the manuscript.

\section{Notes and references}

1 G. Qiu, Z. Gai, Y. Tao, J. Schmitt, G. A. Kullak-Ublick and J. Wang, Dual-functional plasmonic photothermal biosensors for highly accurate severe acute respiratory syndrome coronavirus 2 detection, ACS Nano, 2020, 14, 5268-5277.

2 P. Pokhrel, C. Hu and H. Mao, Detecting the coronavirus (COVID-19), ACS Sens., 2020, 5, 2283-2296. 
3 J. Cao, T. Sun and K. T. V. Grattan, Gold nanorod-based localized surface plasmon resonance biosensors: A review, Sens. Actuators, B, 2014, 195, 332-351.

4 J. Stone, S. Jackson and D. Wright, Biological applications of gold nanorods, Wiley Interdiscip. Rev.: Nanomed. Nanobiotechnol., 2011, 3, 100-109.

5 F. Mazzotta, T. W. Johnson, A. B. Dahlin, J. Shaver, S.-H. Oh and F. Höök, Influence of the evanescent field decay length on the sensitivity of plasmonic nanodisks and nanoholes, ACS Photonics, 2015, 2, 256-262.

6 L. Zheng, G. Cai, W. Qi, S. Wang, M. Wang and J. Lin, Optical biosensor for rapid detection of Salmonella typhimurium based on porous gold@platinum nanocatalysts and a 3D fluidic chip, ACS Sens., 2019, 5, 65-72.

7 L. Guo, J. A. Jackman, H.-H. Yang, P. Chen, N.-J. Cho and D.-H. Kim, Strategies for enhancing the sensitivity of plasmonic nanosensors, Nano Today, 2015, 10, 213-239.

8 N. Zhang, Y. J. Liu, J. Yang, X. Su, J. Deng, C. C. Chum, M. Hong and J. Teng, High sensitivity molecule detection by plasmonic nanoantennas with selective binding at electromagnetic hotspots, Nanoscale, 2014, 6, 1416-1422.

9 D. Gu, C. Zhang, Y.-K. Wu and L. J. Guo, Ultrasmooth and thermally stable silver-based thin films with subnanometer roughness by aluminum doping, ACS Nano, 2014, 8, 10343-10351.

10 Z. Cheng, R. Wang, Y. Xing, L. Zhao, J. Choo and F. Yu, SERS-based immunoassay using gold-patterned array chips for rapid and sensitive detection of dual cardiac biomarkers, Analyst, 2019, 144, 6533-6540.

11 W. Chen, M. D. Thoreson, S. Ishii, A. V. Kildishev and V. M. Shalaev, Ultra-thin ultra-smooth and low-loss silver films on a germanium wetting layer, Opt. Express, 2010, 18, 5124-5134.

12 L. J. Sherry, S.-H. Chang, G. C. Schatz, R. P. Van Duyne, B. J. Wiley and Y. Xia, Localized surface plasmon resonance spectroscopy of single silver nanocubes, Nano Lett., 2005, 5, 2034-2038.

13 B. Päivänranta, H. Merbold, R. Giannini, L. Büchi, S. Gorelick, C. David, J. F. Löffler, T. Feurer and Y. Ekinci, High aspect ratio plasmonic nanostructures for sensing applications, ACS Nano, 2011, 5, 6374-6382.

14 S. Zeng, D. Baillargeat, H.-P. Ho and K.-T. Yong, Nanomaterials enhanced surface plasmon resonance for biological and chemical sensing applications, Chem. Soc. Rev., 2014, 43, 3426-3452.

15 C.-Y. Tsai, K.-H. Chang, C.-Y. Wu and P.-T. Lee, The aspect ratio effect on plasmonic properties and biosensing of bonding mode in gold elliptical nanoring arrays, Opt. Express, 2013, 21, 14090-14096.

16 A. S. Gangnaik, Y. M. Georgiev and J. D. Holmes, New generation electron beam resists: a review, Chem. Mater., 2017, 29, 1898-1917.

17 H. Shahali, J. Hasan, H. Wang, T. Tesfamichael, C. Yan and P. K. D. V. Yarlagadda, Evaluation of particle beam lithography for fabrication of metallic nano-structures, Procedia Manuf., 2019, 30, 261-267.
18 S. Fruncillo, X. Su, H. Liu and L. S. Wong, Lithographic processes for the scalable fabrication of micro-and nanostructures for biochips and biosensors, ACS Sens., 2021, 6, 2002-2024.

19 Y. Chen, Nanofabrication by electron beam lithography and its applications: A review, Microelectron. Eng., 2015, 135, 57-72.

20 C.-W. Kuo, J.-Y. Shiu, Y.-H. Cho and P. Chen, Fabrication of large-area periodic nanopillar arrays for nanoimprint lithography using polymer colloid masks, Adv. Mater., 2003, 15, 1065-1068.

21 J. Chen, Q. Ma, X. J. Wu, L. Li, J. Liu and H. Zhang, Wetchemical synthesis and applications of semiconductor nanomaterial-based epitaxial heterostructures, Nano-Micro Lett., 2019, 11(1), 1-28.

22 M. Wang, X. Ye and J. Feng, Fabrication of length-controlled polymer nanopillars using poly(dimethylsiloxane) filled anodised aluminium oxide templates, Micro Nano Lett., 2013, 8, 713-717.

23 E. Hoque, J. A. DeRose, G. Kulik, P. Hoffmann, H. J. Mathieu and B. Bhushan, Alkylphosphonate modified aluminum oxide surfaces, J. Phys. Chem. B, 2006, 110, 10855-10861.

24 J. Liu, Y. Ma, J. Shao, S. Zhang and Y. Chen, Ultra-tall subwavelength gold nano pillars for high sensitive LSPR sensors, Microelectron. Eng., 2018, 196, 7-12.

25 D. Zhang, S. Misra, L. Li, X. Wang, J. Jian, P. Lu, X. Gao, X. Sun, Z. Qi and M. Kalaswad, Tunable optical properties in self-assembled oxide-metal hybrid thin films via Au-phase geometry control: from nanopillars to nanodisks, Adv. Opt. Mater., 2020, 8, 1901359.

26 S. G. Higgins, M. Becce, A. Belessiotis-Richards, H. Seong, J. E. Sero and M. M. Stevens, High-aspect-ratio nanostructured surfaces as biological metamaterials, Adv. Mater., 2020, 32, 903862.

27 S. S. Aćimović, H. Špová, G. Emilsson, A. B. Dahlin, T. J. Antosiewicz and M. Käll, Superior LSPR substrates based on electromagnetic decoupling for on-a-chip high-throughput label-free biosensing, Light: Sci. Appl., 2017, 6, e17042.

28 K. A. Willets and R. P. Van Duyne, Localized surface plasmon resonance spectroscopy and sensing, Annu. Rev. Phys. Chem., 2007, 58, 267-297.

29 S. Celiksoy, W. Ye, R. Ahijado-Guzmán and C. Sönnichsen, Single out-of-resonance dielectric nanoparticles as molecular sensors, textit, ACS Sens., 2021, 6, 716-721.

30 K. M. Mayer, S. Lee, H. Liao, B. C. Rostro, A. Fuentes, P. T. Scully, C. L. Nehl and J. H. Hafner, A label-free immunoassay based upon localized surface plasmon resonance of gold nanorods, ACS Nano, 2008, 2, 687-692.

31 E. Mauriz, P. Dey and L. M. Lechuga, Advances in nanoplasmonic biosensors for clinical applications, Analyst, 2019, 144, 7105-7129.

32 J. A. Jackman, A. R. Ferhan and N.-J. Cho, Nanoplasmonic sensors for biointerfacial science, Chem. Soc. Rev., 2017, 46, 3615-3660.

33 L. Soares, A. Csáki, J. Jatschka, W. Fritzsche, O. Flores, R. Franco and E. Pereira, Localized surface plasmon resonance 
(LSPR) biosensing using gold nanotriangles: detection of DNA hybridization events at room temperature, Analyst, 2014, 139, 4964-4973.

34 X. Ren, X. Meng and F. Tang, Preparation of Ag-Au nanoparticle and its application to glucose biosensor, Sens. Actuators, B, 2005, 110, 358-363.

35 S. M. Novikov, C. Frydendahl, J. Beermann, V. A. Zenin, N. Stenger, V. Coello, N. A. Mortensen and S. I. Bozhevolnyi, White light generation and anisotropic damage in gold films near percolation threshold, ACS Photonics, 2017, 4, 1207-1215.

36 L. P. F. Peixoto, J. F. L. Santos and G. F. S. Andrade, Anal. Chim. Acta, 2019, 1084, 71-77.

37 S.-W. Lee, K.-S. Lee, J. Ahn, J.-J. Lee, M.-G. Kim and Y.-B. Shin, Highly sensitive biosensing using arrays of plasmonic $\mathrm{Au}$ nanodisks realized by nanoimprint lithography, ACS Nano, 2011, 5, 897-904.

38 K. C. Ng and W. Cheng, Fine-tuning longitudinal plasmon resonances of nanorods by thermal reshaping in aqueous media, Nanotechnology, 2012, 23, 105602.

39 X. Peng, Y. Zhou, K. Nie, F. Zhou, Y. Yuan, J. Song and J. Qu, Promising near-infrared plasmonic biosensor employed for specific detection of SARS-CoV-2 and its spike glycoprotein, New J. Phys., 2020, 22, 103046.

40 D. Kim, E. Choi, C. Lee, Y. Choi, H. Kim, T. Yu and Y. Piao, Highly sensitive and selective visual detection of $\mathrm{Cr}(\mathrm{vI})$ ions based on etching of silver-coated gold nanorods, Nano Convergence, 2019, 6, 1-9.

41 N. A. Joy, B. K. Janiszewski, S. Novak, T. W. Johnson, S.-H. Oh, A. Raghunathan, J. Hartley and M. A. Carpenter, Thermal stability of gold nanorods for high-temperature plasmonic sensing, J. Phys. Chem. C, 2013, 117, 11718-11724.

42 S. Devaraj, U. Singh and I. Jialal, Human C-reactive protein and the metabolic syndrome, Curr. Opin. Lipidol., 2009, 20, 182.
43 D. Thompson, M. B. Pepys and S. P. Wood, The physiological structure of human C-reactive protein and its complex with phosphocholine, Structure, 1999, 7, 169-177.

44 S. F. Pedersen and Y.-C. Ho, et al., J. Clin. Invest., 2020, 130, 2202-2205.

45 K. G. Andersen, A. Rambaut, W. I. Lipkin, E. C. Holmes and R. F. Garry, The proximal origin of SARS-CoV-2, Nat. Med., 2020, 26, 450-452.

46 Y. Liu, L. Zhan, Z. Qin, J. Sackrison and J. C. Bischof, Ultrasensitive and highly specific lateral flow assays for point-of-care diagnosis, ACS Nano, 2021, 15, 3593-3611.

47 A. Garifullina and A. Q. Shen, Optimized immobilization of biomolecules on nonspherical gold nanostructures for efficient localized surface plasmon resonance biosensing, Anal. Chem., 2019, 91, 15090-15098.

48 K. Chen, Y. Xu, S. Rana, O. R. Miranda, P. L. Dubin, V. M. Rotello, L. Sun and X. Guo, Electrostatic selectivity in protein-nanoparticle interactions, Biomacromolecules, 2011, 12, 2552-2561.

49 J. Seaberg, H. Montazerian, M. N. Hossen, R. Bhattacharya, A. Khademhosseini and P. Mukherjee, Hybrid nanosystems for biomedical applications, ACS Nano, 2021, 15, 2099-2142.

50 A. Shrivastava and B. Gupta, Methods for the determination of limit of detection and limit of quantitation of the analytical methods, Chron. Young Sci., 2011, 2, 21.

51 X. Xu, X. Liu, Y. Li and Y. Ying, A simple and rapid optical biosensor for detection of aflatoxin B1 based on competitive dispersion of gold nanorods, Biosens. Bioelectron., 2013, 47, 361-367.

52 T. Ji, Z. Liu, G. Wang, X. Guo, C. Lai, H. Chen, S. Huang, S. Xia, B. Chen and H. Jia, Detection of COVID-19: A review of the current literature and future perspectives, Biosens. Bioelectron., 2020, 166, 112455. 